\title{
VARIA
}

\section{FORMATION À DISTANCE ET COMMUNICATION MÉDIATISÉE ${ }^{1}$}

\author{
Daniel Peraya
}

La Communauté européenne assure aujourd'hui un rôle essentiel dans le développement de la formation à distance (FAD) non seulement au sein des États membres mais aussi dans les pays de l'AELE, en Europe centrale et orientale. Rappelons que depuis 1987, le Parlement européen a reconnu l'importance de l'apprentissage ouvert et à distance à l'occasion d'une résolution concernant les universités ouvertes dans la $\mathrm{CE}^{2}$. A la suite de celle-ci, la Commission des

1 Cet article constitue une version remaniée de lintroduction au rapport final $L a$ conception et la production du matériel d'enseignement à distance à la FernUniversität et à l'Open Universiteit réalisé, sous mandat de l'Office fédéral pour l'éducation et la science (Berne). Ce rapport constitue l'un des aspects de la participation suisse à un projet de développement d'un centre de formation à distance à Budapest (Projet Fernstudienzentrum Budapest, Programme européen TEMPUS) en collaboration avec les universités à distance de Hagen, de Heerlen et de Vienne. L'enquête sur le terrain, le dépouillement de l'information et le travail rédactionnel ont été réalisés en collaboration avec $\mathrm{Cl}$. Hässig, assistant à TECFA et chargé de projets auprès du Centre Européen de la Culture (Genève). Daniel Peraya est Maître d'Enseignement et de Recherche à l'Université de Genève et Chargé de cours invité au Département de communication de l'Université catholique de Louvain.

2 Parlement Européen, Résolution sur les universités ouvertes dans la Communauté européenne, Document A 2-69/87, 10 juillet 1987.

Recherches en communication, $\mathrm{n}^{\circ} 1$ (1994). 
Communautés européennes a approuvé un rapport sur l'enseignement ouvert à distance dans la Communauté1. Enfin, en novembre 1992, était publié le Mémorandum sur l'apprentissage ouvert et à distance ${ }^{2}$ dont l'objectif était de montrer l'importance de ce mode d'apprentissage pour les États membres de la CE. Quant aux associations nationales, européennes (AEUED, SATURN, EDEN, etc.) et internationales (ICDL, ICDE), elles connaissent un développement sans précédent.

Mais il est un autre ordre de raisons qui motive notre intérêt pour ce mode de formation. Nous croyons en effet que de par ses caractéristiques propres ${ }^{3}$, la FAD peut être l'occasion d'une clarification méthodologique essentielle dont la formation présentielle sera, par un effet de retour, la première à bénéficier. La rupture entre les actes d'enseignement et d'apprentissage, l'isolement de l'apprenant, la conception modulaire des unités d'enseignement, etc. nécessitent de la part de l'ensemble des personnes impliquées dans la formation -l'enseignant, le concepteur médiatique, le technologue de l'éducation, etc.- une explicitation exhaustive de la démarche pédagogique : définition des objectifs; sélection, structuration et accessibilité des contenus, aide à l'apprentissage, médiatisation des contenus d'enseignement, choix des médias et création d'un environnement d'apprentissage, etc. On réalise d'ailleurs rarement que la formation présentielle inclut des séquences d'apprentissage dont les caractéristiques sont identiques à celles de la FAD: l'utilisation de didacticiels en autoformation, les devoirs à domicile, la préparation aux examens, etc.

Enseigner à distance, c'est en effet enseigner en différé. C'est en conséquence enseigner à travers la médiation de supports de communication puisque les contenus d'enseignement, les exercices, les consignes de travail, etc. ne peuvent être transmis à l'apprenant que par l'intermédiaire de moyens d'information et de communication: documents écrits, supports audiovisuels classiques ou informatisés, nouvelles technologies de la communication et de l'information (NTCI). Aujourd'hui l'évocation la plus fréquente est celle d'un pont

1 CCE, Enseignement supérieur ouvert et à distance dans la Communauté européenne, Rapport de la Commisssion, SEC (91) 897 final, 24 mai 1991.

2 CCE, Mémorandum sur l'apprentissage ouvert et à distance dans la Communauté européenne, COM(91) 338 final, 12 novembre 1991.

3 Voir par exemple Fr. HENRI et A. KAYE, Le savoir à domicile, Montréal, Les presses de l'Université du Québec, 1985, Télé-université ou D. PERAYA, L'enseignement à distance. Vers de nouvelles perspectives, Genève, Département de l'instruction publique, 1990. 
médiatique: celui-ci offrirait "un ensemble de moyens d'autoformation ou d'information combinant l'emploi des divers médias selon leur spécificité -avec l'utilisation de documents imprimés et audiovisuels, de matériel de travaux pratiques, travail en groupe et usage de la télématique"1. Les technologies centrales de ce type de projet sont les NTCI, essentiellement le Minitel, la communication médiatisée par ordinateur (Computer Mediated Communication, CMC), le courrier et les conférences électroniques ${ }^{2}$.

Voilà qui ne devrait pas surprendre. Vaincre la distance a en effet été la vocation des premières institutions d'enseignement à distance. Ce mode de formation s'adressait en effet aux apprenants qui, isolés ou trop éloignés des centres d'apprentissage ou des écoles, ne pouvaient avoir accès à l'instruction classique et à l'enseignement présentiel $^{3}$. Aussi les moyens de communication et de diffusion ont-ils toujours occupé un rôle central dans ce mode de formation: la poste bien sûr, qui en a été le premier vecteur et plus tard le téléphone ou la radiotélévision ${ }^{4}$. Historiquement, on considère que le véritable développement de la FAD a été rendu possible au siècle dernier grâce à l'invention du timbre-poste, à la généralisation de services postaux bon marché ainsi qu'à la découverte d'un mode de production d'un papier bon marché et de bonne qualité. Autrement dit, l'essor de la FAD est lié à la transformation des modes de circulation, de transmission et de stockage de l'information ${ }^{5}$. Les cours par correspondance proposés par Pitman dès 1870 en sont la meilleure illustration puisqu'ils se sont développés à l'époque de ces innovations. Rappeler

1 GAUTHIER in CNED, Eléments pour la connaissance de l'enseignement à distance, Vanves, Cahier d'information du centre de Vanves, $\mathrm{n}^{\circ} 1,1987$.

2 Le développement des télécommunications, l'accroissement de la vitesse de transmission et de la capacité des réseaux banaliseront, à terme, le transfert d'images y compris animées.

3 Tel était par exemple l'objectif des premiers cours par correspondance: dès 1914 à Melbourne, 1919 à Vancouver ou encore dès 1922, en Nouvelle Zélande. Des motivations du même ordre ont présidé à la création du CNED en France au moment de la seconde guerre mondiale: permettre que les élèves éloignés de leur lycée d'origine ou disséminés par l'exode continuent leurs études. Enfin, le développement de l'enseignement à distance dans des pays tels que la Chine, l'Inde ou l'Australie montre bien que la volonté de réduire, voire d'annuler, la distance géographique demeure encore une composante non négligeable de la formation à distance.

${ }^{4}$ La radiotéléphonie a été utilisée assez rapidement pour joindre les élèves isolés, par exemple dans le bush australien dès 1942. Rappelons la dénomination anglaise d'“Université des airs".

5 Fr. HENRY et A. KAYE, op. cit. 
à ce propos que l'on a parfois désigné les institutions d'enseignement à distance classiques -les instituts d'enseignement par correspondance des services publics- comme la "conjonction d'une école et d'un bureau de poste"l n'a donc rien d'une boutade: à la FernUniversität de Hagen, par exemple, les frais d'envois postaux représentent un budget de trois millions de marks par an pour les 50.000 étudiants ce qui correspond à 300.000 envois, en moyenne par mois.

On ne devrait pas s'étonner que la FAD connaisse actuellement un renouveau important alors que les télécommunications sont en plein essor et que la télé-informatique bouleverse entièrement notre façon de conserver, d'exploiter, de diffuser et de faire circuler l'information : la télématique, le téléchargement, la vidéoconférence, la communication assistée par ordinateur, le multimédia apparaissent comme autant de technologies qui devraient progressivement succéder au timbre poste et au papier bon marché. Et la comparaison semble fondée car ce qui se dessine aujourd'hui c'est bien une mutation des moyens de communication, de circulation et de diffusion de l'information ainsi que des formes de stockage -de conservation et d'accès- de cette même information. L'informatique qui offre la possibilité de conserver sous un format unique -un fichier numériquetoutes formes d'information (textes, données chiffrées, images fixes ou en mouvement, sons) décuple en effet les possibilités de communication, d'interaction et de téléprésence. La télé-informatique constitue quant à elle, la clé de la déscolarisation, de la délocalisation tant géographique que temporelle. C'est donc à raison que l'on a pu dire que ces nouvelles technologies permettraient une meilleure intégration de la formation à la vie professionnelle, une occupation des temps morts de la vie sociale, des temps "poreux"2.

Les universités américaines utilisent depuis longtemps les formes de communication électronique pour l'encadrement de leurs étudiants et les évaluations montrent que c'est en général, à la satisfaction des usagers $^{3}$. Citons aussi l'Open University qui, dans le cadre d'un cours

1 L'expression est de Fr. Duchesnes, Inspecteur auprès du Service de l'Enseignement à distance de la Communauté française de Belgique.

2 A.A. Moles, Théorie structurale de la communication et société, Paris, CNET/ENST, Masson, 1988.

3 Cf. quelques ouvrages récents: M. RAPAPORT, Computer Mediated Communication, New York, John Wiley \& Sons, Inc., 1991; R. MASON, A. KAYE, Mindweave, Oxford, Pergamon press, 1989; M.D. WAGGONER, Empowering Networks. Computer conferencing in Education, New Jersey, Educational Technology Publications, Englewood Cliffs, 1992. 
sur les technologies, développe depuis 1987 le système de communication COSY, véritable campus électronique: le système permet donc d'assurer à distance certains enseignements, l'encadrement et le suivi pédagogiques, la communication enseignant-enseignés et enseignésenseignés, enfin l'accès aux ressources documentaires. Quant aux universités européennes, elle participent en général à des grands réseaux de communication et d'information transnationaux et transcontinentaux: le courrier électronique et les forums sont pratiques courantes ${ }^{1}$.

Nous serions donc entrés dans l'ère des télécommunications : le village électronique de Mac Luhan, Emerec ${ }^{2}$, la "société communicationnelle" ne sont déjà plus tout à fait une utopie. Mais cette société communicationnelle se veut en même temps (in)-formationnelle et éducative.

Le développement de ces technologies pour la FAD ne se fait pourtant pas sans difficulté et les controverses soulevées à cette occasion rappellent à s'y méprendre la problématique de l'introduction de l'audiovisuel dans l'enseignement, dans les années '70. On observe une fois encore la même inadéquation entre le discours zélateur des inventeurs ${ }^{3}$ et les réalités pédagogiques. C'est que les inventeurs repensent la société à partir de leurs inventions et de la place qu'elle devraient, selon eux, occuper. Or cette conception, dans le domaine de l'innovation pédagogique, se traduit le plus souvent par ces trois postulats: utilité, universalité et supériorité selon lesquels tout ce qui est nouveau est mieux que ce qui a précédé et qui, du même coup, est présenté de façon réductrice ${ }^{4}$.

S'ajoutent à cette argumentation assez classique, le poids des déterminations économiques et des enjeux que représente le marché des NTCI, ou plus particulièrement aujourd'hui le multimédia. La restructuration de l'économie sous l'effet des technologies, la pénétra-

1 Dans cette perspective, il faut mentionner World-Wide Web, un hypertexte développé par le CERN qui relie des serveurs universitaires à travers le monde.

2 J. ClouTier, La communication audio-scripto-visuelle à l'heure des self-média ou l'ère d'Emerec, Montréal, Presses de l'Université de Montréal, 1973. Dans cette direction, la prodigieuse expansion de la téléphonie mobile et de ses accessoires comme le sémaphon constitue un indice intéressant de l'évolution des usages: aujourd'hui c'est une personne que l'on appelle et celle-ci doit pouvoir être atteignable où qu'elle soit. Jadis, l'usage téléphonique voulait qu'on appelle un lieu pour y contacter une personne.

3 J. Perriault, La logique de l'usage, Paris, Flammarion, 1989.

4 G. JACQUINOT in Fr. HENRI et A. KAYES, op. cit., p. 269. 
tion de celles-ci dans tous les secteurs de la vie professionnelle, la nécessité de développer ou de renouveler un marché déjà partiellement saturé, la concurrence de plus en plus grande entre les firmes, etc. sont autant de facteurs qui facilitent l'émergence de groupes partisans parfois excessifs de l'introduction des NTCI dans l'éducation. Ceux-ci reprennent alors à leur compte ces différents postulats : les entrepreneurs succèdent en effet bien vite aux inventeurs.

Certes, il existe bon nombre d'expériences réussies d'intégration des NTCI dans la FAD, mais leur utilisation ainsi que celle des différents supports multimédias font encore figure d'exception. Comme le soulignait une étude du GREP: “(...) il faut bien convenir qu'à travers la multiplicité des expériences en cours, il apparaît un décalage sensible entre le profil idéal de l'EAD [enseignement à distance], nourri des développements de NTCI, parfois d'évolutions encore à venir et seulement projetées ou demeurées à l'état expérimental, et la plupart des réalisations effectivement rencontrées"'. Par ailleurs, dans la majorité des institutions qui ont développé ces nouveaux environnements d'apprentissage, le support écrit constitue toujours une part importante du temps d'activité de l'apprenant : entre 50 et $80 \%$, selon les experts. Ce n'est donc pas par hasard que l'on dit de l'imprimé qu'il est le "média gagnant" et qu'il le sera vraisemblablement encore longtemps: "Le premier média à constituer reste l'édition d'un ensemble de brochures interactives, dont la fonction sera de transmettre des contenus scientifiques structurés."2. Il suffit pour s'en convaincre d'observer la répartition des différentes activités et la place réservée aux productions multimédias ainsi qu'aux NTCI dans les systèmes de formation à distance actuels. L'Open University propose depuis fort longtemps, à titre de référence, la ventilation moyenne suivante:

- 5 à $10 \%$ de temps-élève en audiovisuel

- $80 \%$ de temps-élève en cours par correspondance;

- $10 \%$ de temps-élève en animation de groupe et autres activités d'encadrement.

1 GREP, Groupe de recherche pour l'Education et la Prospective, Le dispositif d'enseignement public à distance agricole : le C.N.P.R., Paris, 1988, (sous la direction de J. ARDolno avec la collaboration de G. BERGER, H. HARvols et J.M. SECA).

2 M.-J. DudezerT-DELbReIl et J.-P. DudezerT, "Formation multimédia: définition et méthodes", in Education permanente. Que faire des nouveaux médias?, 109-121, Vol. 93/94, p. 112. 
Dans le même ordre d'idée, à l'Open University, les premières analyses du système Cosy montrent que le temps moyen d'utilisation est de 10 heures par étudiant et qu'un tiers des étudiants inscrits ne l'utilise jamais sans qu'il soit toujours possible d'identifier si ces étudiants sont aussi ceux qui abandonnent les cours ${ }^{1}$.

La clé de répartition du temps d'utilisation des médias proposée par l'Open University ne fait pas figure d'exception comme le montre cette seconde proposition adaptée, quant à elle, au contexte français où l'usage du Minitel est très répandu:

- $10 \%$ du temps d'apprentissage en audiovisuel

- $15 \%$ du temps d'apprentissage en EAO;

- $15 \%$ du temps d'apprentissage en télématique;

- $50 \%$ du temps d'apprentissage en cours par correspondance;

- $10 \%$ du temps d'apprentissage en animation de groupe et autres activités conviviales ${ }^{2}$.

Enfin, les propositions d'une structure de formation modulaire pour les brevets français de technicien agricole (BTA) du Centre National de Promotion Rurale faisaient état, pour chaque module de 170 heures, de 25 à 35 heures d'apprentissage sous forme de multimédias ou de didacticiels. Sur l'ensemble des cinq modules analysés par le GREP' ${ }^{3}$, l'opération de médiatisation a porté sur 160 heures d'enseignement sur un total de 730 heures soit $21,9 \%$ répartis comme suit: 80 heures de didacticiels, 45 heures de vidéos et 35 heures de cassettes audio.

Ces différentes formules de répartition des activités d'apprentissage démontrent bien la nécessité d'opérer des choix quant aux différents médias et à leur taux d'utilisation dans une perspective d'utilisation intégrée.

\section{Une taxonomie des médias}

Autrement dit, il s'agit pour le designer pédagogique de pouvoir répondre aux deux questions suivantes: quel média choisir et pourquoi? Parmi les critères souvent considérés comme décisifs, on signa-

1 Communication orale de A. Kayes au DEC-JITOL Evaluation Workshop, September 30-October 1, 1992, Neurope Lab, Archamps.

2 DORLÉANS in CNED (1987), op. cit.

3 GREP, op. cit., p. 100-104. 
lera le contenu et l'objectif de la tâche d'apprentissage ${ }^{1}$ ou encore le projet éducatif: “(...) ce n'est pas le dispositif de formation à distance, avec ou sans médias audiovisuels, qui détermine le projet éducatif et donne le modèle pédagogique sur lequel repose le système mais l'inverse"'. La FAD ne doit donc en aucun cas devenir une vitrine d'exposition technologique où l'agencement des médias et les procédures technologiques de communication deviendraient une fin en soi. En conséquence, nul ne s'étonnera si, du point de vue méthodologique, l'une des priorités consiste en l'analyse des fonctions pédagogiques du processus d'autodidaxie et si c'est par rapport à celles-ci que l'on envisage l'intégration éventuelle des médias et des technologies.

Cette exigence méthodologique conduit inévitablement à poser le problème de la taxonomie des médias. Elle suppose encore que soit résolue la question de la spécificité des différents médias. La création d'une taxonomie opérationnelle des médias devrait permettre de répondre "simplement" à la question suivante: "quel média, pour quels contenus et quel public?" que l'on devrait d'ailleurs spécifier de cette façon: "quel est le meilleur médium ou le plus approprié à l'objectif visé?" ou encore: "quels attributs spécifiques de quel média sont-ils propres à favoriser l'apprentissage en fonction de quels traits caractéristiques de l'enseignement et compte tenu de quelle tâche à accomplir?"3. La question est importante car sa réponse détermine les choix que tous les enseignants, rédacteurs, concepteurs et designers pédagogiques doivent opérer dans leur pratique quotidienne. Mais elle l'est encore car elle est profondément liée à l'efficacité du média dans son usage pédagogique et donc à celle, plus générale, de l'efficacité de l'enseignement à distance autant que présentiel. Il ne faut donc pas s'étonner si ces recherches ont concerné d'abord l'enseignement présentiel et si certaines conclusions valent pour ces deux formes d'enseignement.

A ces questions, il n'existe pas de réponse définitive. Non pas que l'on manque d'études expérimentales: depuis près de quarante ans, on compte un très grand nombre d'analyses et de travaux tant européens qu'américains. Néanmoins aucun résultat n'est apparu réellement significatif: "La plupart d'entre elles (les recherches expérimen-

1 R.M. GAGNÉ, The conditions of learning, New York, Holt, Rinehart and Winston, 1965.

2 G. JACQUINOT in KAYES, op. cit., p. 286.

3 E. U. HEIDT, "La taxonomie des médias", in Communications, Apprendre des médias, n 33, 1987, p. 51-74. 
tales) comparent deux ou plusieurs d'entre eux (les médias) afin d'établir leur efficacité relative. Toutes les tentatives pour tirer à partir de ces éléments des conclusions généralisables ont cependant échoué ou bien il est impossible de dégager des différences statistiquement significatives ou bien les résultats sont contradictoires"1. Quant à la spécificité des médias, les chercheurs en ont débattu longtemps sans arriver à des résultats plus concluants : "La triste réalité cependant demeure que nous ne savons ni mesurer les effets psychologiques des médias, ni les adapter aux buts et aux fonctions de l'école"2. Au-delà des résultats partiels tenus pour acquis aujourd'hui tel le "Picture Superiority Effect" (PSE) ${ }^{3}$ ou l'effet de supplantation ${ }^{4}$, il semble bien que notre connaissance globale des médias demeure insuffisante pour pouvoir développer, sur la base d'une théorie des médias, une analyse des processus d'apprentissage et en conséquence, une taxonomie réellement opérationnelle.

D'autres facteurs, liés à l'analyse de la pratique pédagogique, rendent la construction de taxonomies encore plus délicate : une analyse empirique semble montrer de façon convaincante que les médias présentent un degré important de plurifonctionnalité et que par ailleurs, ils sont en grande partie interchangeables. ${ }^{5}$ Un même document audiovisuel, prenons par exemple le cas de l'iconographie médicale ou dentaire, peut toujours assumer plusieurs fonctions pédagogiques parmi lesquelles le concepteur devra choisir, notamment en fonction

1 On pourra lire une synthèse de ces recherches dans HeIDT, op. cit. et aussi dans M. SCHOLER, La technologie de l'éducation, Montréal, Presses de l'Université de Montréal, 1988.

2 SAETTLER cité par SchOler, op. cit.

3 D. REID, "Quelques investigations sur le rôle des images dans l'apprentissage à partir de textes de biologie", in Cahiers du CRELEF. Les Formes du Savoir dans les manuels scientifiques, $\mathrm{n}^{\circ} 28,1989,1-2, \mathrm{p} .73-100$.

4 Voir par exemple, G. SALOMON, Interaction of Media Cognition and Learning, Jossey-Bass, 1979; L.J. AUSBURN et F.B. AusBuRN, "A supplantation Model for Instructional Design : Investigation of a Behavioural Science Approach", in Australian Journal of Education, octobre 1978, p. 277-294; B.E. LYNCH, "Effects of Selected Filmic Coding Elements of TV on the Development of the Euclidean Concepts of Horizontality and verticality" in Adolescents ERIC ED 267784, 1986; P.L. SMITH, Supplantation versus generative Models Implications for Designers of Instructional Text, 1985; J. DONNAY, Supplantation télévisuelle el communication orale, Thèse de Doctorat, Université de Liège, 1981, non publiée.

5 D. PERAYA, L'utilisation des moyens d'enseignement audiovisuels entre les choix et les contraintes, Document d'information et de formation méthodologique $n^{\circ} 5$, Genève, Commission de l'enseignement du Rectorat, Université de Genève, Janvier 1990. 
de ses objectifs. Des clichés radiologiques dentaires, par exemple, peuvent être utilisés, au moins, pour ces trois différentes fonctions:

a. l'iconographie a toute son importance dans de nombreux cours où la réalité dont traite l'enseignant ne peut être perçue que par la médiation d'une représentation. C'est la fonction vicariale et illustrative des clichés qui est alors sollicitée;

b. ces mêmes images peuvent servir de support à une activité d'observation et d'analyse individuelle ou collective: elles servent donc de document d'apprentissage pour les étudiants en tant qu'elles sont un déclencheur et un support d'activité pour les étudiants;

c. enfin, les mêmes images peuvent encore être utilisées pour évaluer les acquis des étudiants à la fin d'un cours: en tant qu'item de contrôle, chacune de celles-ci pourrait fort bien, par exemple, servir de base à un questionnaire à choix multiple.

Inversement, les différents moyens sont, en partie tout au moins, interchangeables et la substitution d'un moyen à un autre est toujours possible, sans ou avec peu de perte d'efficacité. Aucun moyen ne possède donc une supériorité absolue sur un autre: choisir l'un d'eux revient à lui accorder momentanément une priorité en raison d'une caractéristique que l'on juge particulièrement pertinente dans une situation donnée. C'est ce paradoxe que formulent fort bien DudezertDebreil et Dudezert dans la taxonomie qu'ils proposent sur la base de l'efficacité postulée des médias: “(...) La dernière fonction, illustration et description de la mise en oeuvre des connaissances, peut, quant à elle, être remplie par des cassettes vidéo, des diapositives, ou tout autre média analogue"1.

Un même document de base peut donner lieu à différentes versions sur différents supports: vidéogramme ou film $16 \mathrm{~mm}$, diapositives ou photographies tirées du film, etc. Il faut donc faire la différence entre le document et son traitement -les formes symboliques de représentation- d'une part, et les caractéristiques du canal de transmission d'autre part. Les films qui présentent une information toujours fugace trouvent dans l'utilisation de certaines diapositives un complément adéquat. Par contre, l'arrêt prolongé sur l'image et les fonctions de recherche rapide (search en mode fast forward ou rewind) propres à la technique du magnétoscope ajoutent à l'avantage

1 Op. cit., p. 110. Nous soulignons. 
du film (projection continue) celui de la diapositive (projection image par image) tout en sachant que les conditions perceptives sont alors modifiées. Enfin, en tant que document d'analyse ou en tant qu'item de contrôle, chaque diapositive pourrait être remplacée par une photographie ou par une affiche, selon que le travail exigé des participants est individuel ou collectif. Seule une caractéristique spécifique empiriquement observable, comme la couleur ou le mouvement, permettrait de fonder un choix à condition d'être la seule pertinente pour comprendre les phénomènes médiatisés ou de présenter un rapport direct avec l'apprentissage et la tâche à accomplir: "l'utilisation du mouvement ne semble efficace que lorsque le contenu particulier de l'apprentissage consiste dans le mouvement et ses caractéristiques ou quand le contenu est renforcé et différencié grâce aux effets produits par l'action en mouvement"'. C'est ce type de critères qui est proposé, par exemple, par l'Open Universiteit (cf. ci-dessous).

Enfin, disons que la diversité des appellations -nouvelles technologies de la communication et de l'information (NTCI), nouvelles technologies de la formation (NTF), etc.- s'applique à une pléiade d'objets techniques dont les fonctionnalités sont de fait très différentes: la télévision DBS et la télévision haute définition ; les téléconférences interactives (image et son); la télématique, le vidéotex, le réseau numérique à intégration de services (RNIS/LSDN), les différentes formes de téléchargement, de consultation et de transaction à distance; dans le domaine de l'image, l'appareil photographique à support magnétique numérisé, le vidéodisque, le CD-Rom, le CD-Worm, le CD-I et DVI, les mondes virtuels, etc. De plus, si certaines technologies modifient profondément les modes de traitement de l'information ainsi que la culture technique de l'usager, par exemple l'informatique et l'image numérisée, d'autres n'exercent que très peu d'influence. De nombreuses innovations technologiques demeurent en effet "cachées" et sont, dans le langage des informaticiens, "transparentes": nos communications téléphoniques, par exemple, sont aujourd'hui le plus souvent transmises sous une forme numérique sans que soient pour autant modifiées les communications vocales ${ }^{2}$. Il n'est donc pas démontré que l'on puisse, à travers une telle diversité de domaines, d'objets techniques et d'usages, découvrir un ensemble de

1 R.W. SPANGENBERG, The motion variable in procedural learning, in AVCR 4, 419 436, 1973, (p. 435), cité par HEIDT, op. cit.

2 Il est clair cependant que certains services informatifs ou transactionnels ne sont possibles qu'à cette condition. 
caractéristiques semblables, de traits spécifiques permettant de fonder un modèle taxonomique général.

\section{Critères intrinsèques et contextuels}

Il n'est pas dans notre intention de présenter les différentes taxonomies existantes : elles sont nombreuses et ce travail critique a déjà été fait ${ }^{1}$. Rappelons simplement que l'une des premières taxonomies pour la FAD -sans doute aussi l'une des plus connues- est celle de Bates, pour la télévision, la radio et les cassettes audio ${ }^{2}$. Citons quelques exemples plus proches de nos préoccupations et commentons-les brièvement.

A l'Open Universiteit de Heerlen, le choix des médias est essentiellement déterminé par les caractéristiques propres au mode de représentation et de traitement de l'information et par les implications cognitives de celles-ci. Ce critère qui s'accorde avec les développements des théories psychologiques et pédagogiques actuelles nous paraît essentiel mais il demanderait de réels développements ${ }^{3}$ :

a. le matériel écrit est choisi soit pour les caractéristiques cognitives du langage verbal ou pour des raisons d'ordre pratique : "Operations on knowledge are only possible with written materials, if they are effectively elicited by questions and assignments, Or if the necessary cognitive operations are already available to the students in some rudimentary form, or can be displayed or described in print in such a way that the student can acquire them.";

b. le matériel audio est considéré comme "an indispensable supplement when aspects of verbal utterance are not sufficiently clear in written material e.g. tone of voice, reading a poem, and when the spoken langage has not to be used to demonstrate a manner of thinkin or speaking in action";

1 Voir HEIDT, op. cit.

2 A.W. BATES, Appropriate Teaching Functions for Television, Radio and Audiocassettes in Open University Courses, in IET Papers on Broadcasting, $\mathrm{n}^{\circ}$ 124, 1974. On lira aussi A.W BATES (ed.), Media and Technology in European Distance Education. Proceeding of the EADTV Workshop on Media, Methods and Technology, Heerlen, EADTU, 1990.

3 G. VAN ENCKEVORT, Distance Higher Education The international Dimension, rapport au Working Committee Distance Education of the German Science Council, Heerlen, Open Universiteit, 1992. Ces critères sont cités d'après le rapport On the design of an Open University (1983). 
c. le matériel vidéo est lui aussi considéré comme un supplément indispensable au langage verbal "when processes or phenomena are themselves subject of instruction and a vicarious experience must be offered; when processes or phenomena through being dynamic and complex cannot be described adequately in words or still picture; and when processes or phenomena described in words do not tie up adequately with the students' experiential word";

d. l'ordinateur enfin, est indispensable lorsqu'il est lui-même objet de l'enseignement, quand il permet la simulation de processus complexes ; bref il s'impose par sa puissance de calcul, sa capacité à exécuter des tâches, aussi diverses soient-elles, ou encore parce qu'il permet une certaine interactivité;

e. les séances de regroupement présentielles: elle seront réduites au minimum en raison des coûts et cette contrainte nous rappelle que, à l'heure des NTCI et de la téléprésence, l'homme communicant devient aussi un homme faiblement rencontrant ${ }^{1}$.

Par rapport au point de vue défendu au début des années '70, par exemple par Spangenberg (cf. ici même, note X, p. YYY), cette taxonomie ne paraît guère novatrice. Pourtant elle est significative d'un courant qu'a pris la recherche dans les années '80. En indiquant que le choix d'un média est le résultat d'un démarche pragmatique prenant en compte d'une part, les aspects médiatiques -les critères méthodologiques ou "intrinsèques"- et d'autre part, les aspects économiques, cette taxonomie fait place aux critères contextuels ou "extrinsèques". Mais il en est d'autres tels que le projet éducatif et les objectifs pédagogiques, l'usage social des médias -le degré de "littéracie" disent les québecois-, les besoins et les modes de consommation culturelle tant des apprenants que des enseignants. Autant de facteurs d'ajustement de la pratique qui seront progressivement pris en compte.

\section{Le cas de l'ordinateur}

Dernières venues dans l'arsenal pédagogique de la $\mathrm{FAD}$, les technologies informatiques posent aux pédagogues et aux concepteurs des problèmes de classification du même ordre que les médias clas-

$1 \mathrm{Ph}$. Breton, "Retour vers le futur", in Autrement. Technologies du quotidien, $\mathrm{n}^{\circ} 3$, mars 1992. 
siques. A titre d'exemple, citons la taxonomie proposée par Chacón pour l'ordinateur ${ }^{1}$. En prenant pour hypothèse initiale que l'ordinateur prolonge certaines capacités humaines, l'auteur attribue à cette technologie trois fonctions générales: traitement de l'information, moyen d'interactivité ${ }^{2}$ et instrument de communication ${ }^{3}$.

a. Traitement de l'information (Information processing): l'ordinateur est utilisé comme instrument professionnel pour exécuter des tâches traditionnellement imputées à l'intelligence humaine telles que le calcul, le tri, l'établissement de relations entres des données, la gestion, le secrétariat, etc. La machine est considérée strictement comme un outil professionnel -gestion, secrétariat, production industrielle, etc.-, comme "a tool in order to accomplish this tasks more efficiently".

b. Moyen d'interaction: il s'agit d'une forme de communication homme-machine, de la capacité à exercer une influence réciproque sur un objet ou une autre personne sans qu'il y ait une véritable communication interpersonnelle puisque la signification du message n'est pas mise en cause. En effet: "Usually human interaction involves communication or exchange of meanings through messages" 4 .

1 F. J. Chacón, A taxonomy of Computer media in Distance Education, Hagen, Zentrales Institut für Fernstudienforshung, Fern Universität, 1991. Les citations cidessous sont extraites des pages 2-3.

2 Un courant francophone voudrait réserver le terme interactif à la communication homme - machine et le terme interactionnel à l'interaction humaine: on pourrait s'attendre en effet à lire interactionnel. Le thème de l'interactivité et de l'interaction a été longuement débattu: on se reportera, par exemple, au Bulletin de IDATE, Interactivité(s), Juillet $1985, \mathrm{n}^{\circ} 20$.

3 Il est intéressant de remarquer que cette tripartition correspond globalement à celle que nous avons eu l'occasion d'observer lors d'une enquête menée auprès de services de formation d'institutions publiques et d'entreprises privées du Canton de Genève à propos de l'usage des NTCI. Cette enquête faisait apparaître que ces technologies étaient utilisées fréquemment en tant qu'outils professionnels, plus rarement en tant qu'outils de communication et ponctuellement en tant qu'outils de formation. Cf. Commission Télé-enseignement du DIP (1991), Rapport final de la Commission Télé-enseignement du DIP, Genève. Ce rapport constitue l'étude d'opportunité réalisée à la demande du Grand Conseil en réponse à une motion de MM. Ph. Fontaine et R. Della Santa, en date du 18 mai 1988.

4 Considérer la communication comme une transmission d'une expérience vicariale ayant pour effet la modification de l'état de de connaissances du destinataire permet de considérer l'interaction homme-machine comme une forme réelle de communication puisque l'état des connaissances - les informations stockées dans la mémoire de la machine- sont évidemment modifiées par l'intervention du 
c. Instrument de communication ${ }^{1}$ : il s'agit ici d'une réelle communication interpersonnelle dont la signification porte sur un contexte référentiel et/ou sur les personnes elles-mêmes."The abilities of computers for encoding and transmitting information, plus their capacity for coordinating many files and inpout outpout devices are used for the purpose of streamlining interpersonal communication".

Sur cette base, Chacón propose alors la taxonomie générale suivante:

TYPE DE MÉDIAS

\begin{tabular}{|c|c|c|c|c|}
\hline $\begin{array}{l}\text { Traitement de } \\
\text { l'information }\end{array}$ & $\begin{array}{l}\text { Calcul } \\
\text { Traitement de } \\
\text { texte } \\
\text { Dessin et } \\
\text { graphisme } \\
\text { Gestion de bases } \\
\text { de données }\end{array}$ & $\begin{array}{l}\text { Desktop } \\
\text { publishing } \\
\text { Extended } \\
\text { worksheets }\end{array}$ & & Système intégré \\
\hline Interactivité & $\begin{array}{l}\text { Évaluation } \\
\text { assistée par } \\
\text { ordinateur }\end{array}$ & $\begin{array}{l}\text { EAO } \\
\text { CBT } \\
\text { etc. }\end{array}$ & $\begin{array}{l}\text { Vidéo interactive } \\
\text { Hypermédia }\end{array}$ & $\begin{array}{l}\text { Multimédia } \\
\text { interactif et } \\
\text { intelligent }\end{array}$ \\
\hline Communication & $\begin{array}{l}\text { Information } \\
\text { bancaire } \\
\text { interactive }\end{array}$ & $\begin{array}{l}\text { Courrier } \\
\text { électronique }\end{array}$ & $\begin{array}{l}\text { Conférence } \\
\text { électronique } \\
\text { CMC }\end{array}$ & CMC Multimédia \\
\hline
\end{tabular}

Source: Chacón, op. cit.

\section{Les formes de communication}

Une autre tentative de classification des nouvelles technologies, destinée au départ à analyser les modalités d'encadrement d'apprenants dans un centre d'autoformation, prend pour critère les différentes modalités de communication pédagogique tuteur-apprenant(s) et le degré d'interactivité de celle-ci, définis à travers certaines

destinateur (voir à ce propos A.A. MoLEs, Théorie structurale de la communication et société, Masson, 1988).

1 D. PERAYA, Rapport pour la création d'une centre d'autoformation, Bruxelles, DG IX, CCE et (1991) Notes du Cours E7, Supports et moyens de formation : les langages audiovisuel, communication socio-educative et didactique, TECFA, FPSE, 1989. 
variables caractéristiques. L'expérience a montré qu'un tel instrument peut aider le formateur à mieux définir la situation d'apprentissage et ses stratégies d'intervention.

\section{La relation tuteur/apprenant}

a. individuelle vs collective:

- individuelle (I): le bénéficiaire de l'assistance est un apprenant strictement identifié et la relation est dès lors personnalisée; par exemple, l'encadrement téléphonique ou un logiciel éducatif ;

- collective (C): la communication s'adresse globalement à un groupe ou : même quand elle est individualisée, elle est mise en commun et chaque membre du groupe peut y avoir accès; par exemple, les séances de regroupement ou les conférences électroniques.

b. synchronique vs asynchronique:

- synchronique (S) : elle se déroule en temps réel; par exemple, la communication téléphonique;

- asynchronique (A) : elle se déroule en temps différé, un délai est nécessaire entre l'émission et la réponse; par exemple, le courrier postal ou électronique.

c. directe vs médiatisée:

- directe (D): la transmission entre le tuteur et l'(es) apprenant(s) n'est médiatisée par aucun canal de communication ; par exemple, les rencontres en face à face qui constituent l'exception dans le cadre de la FAD;

- médiatisée (M): la transmission recourt à un canal de communication conventionnel ou technologique, ce qui est par définition, le cas de la FAD.

Ces trois variables caractéristiques peuvent être représentées dans une matrice tridimensionnelle (cf. schémas 1 et 2 ci-dessous), celle-ci permettant alors de positionner chacun des moyens d'assistance choisi tout en mettant en évidence leurs relations mutuelles, leur degré de ressemblance ou de proximité. Dans cette matrice:

a. le plan de symétrie avant - arrière permet d'identifier l'opposition individuelle - collective (I - C);

b. le plan de symétrie gauche - droite permet d'identifier l'opposition synchrone/asynchrone ( $\mathrm{S}-\mathrm{A}$ );

c. le plan de symétrie bas - haut permet d'identifier l'opposition directe/médiatisée (D - M). 


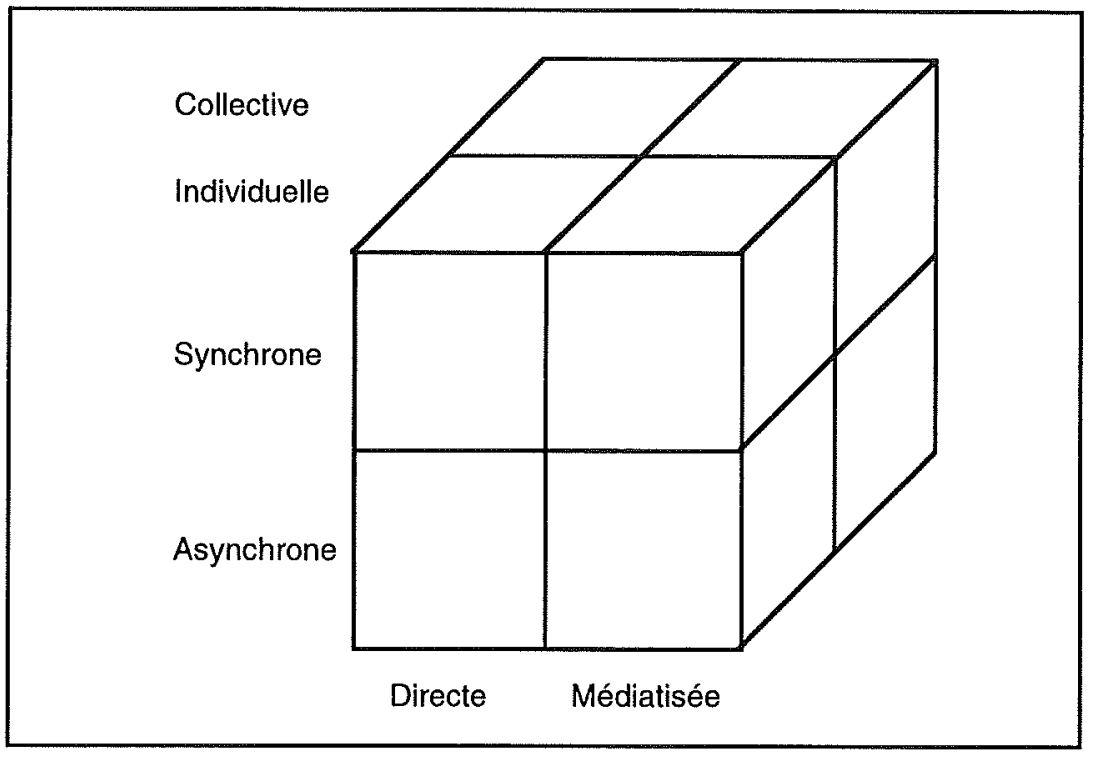

\section{Schéma 1: Matrice tridimensionnelle d'analyse des formes de communication pédagogique}

\section{Le degré d'interactivité1}

Le degré d'interactivité de la communication dépend de la qualité de l'échange entre le tuteur et l'(es) apprenant(s), mais il demeure très difficile à évaluer:

a. la directionnalité de l'échange:

- certains moyens sont unidirectionnels: ils ne permettent qu'une communication à sens unique et leur interactivité est faible ;

- d'autres sont bi- ou multidirectionnels: certaines formes de vidéoconférences, par exemple, sont unidirectionnelles au niveau de l'image (on ne peut jamais voir que le tuteur), mais pluridirectionnelles au niveau du son (chaque apprenant peut lui parler tandis qu'il peut répondre à chacun); la tendance actuelle est de favoriser les communications multidirectionnelles image et son;

1 Si l'on s'en tient à ces différents critères, on se rend compte aisément qu'un cours traditionnel ou qu'une conférence peuvent être moins interactifs qu'un logiciel éducatif bien construit. L'appréciation du degré d'interactivité est donc toujours délicate et il faut procéder au cas par cas. 


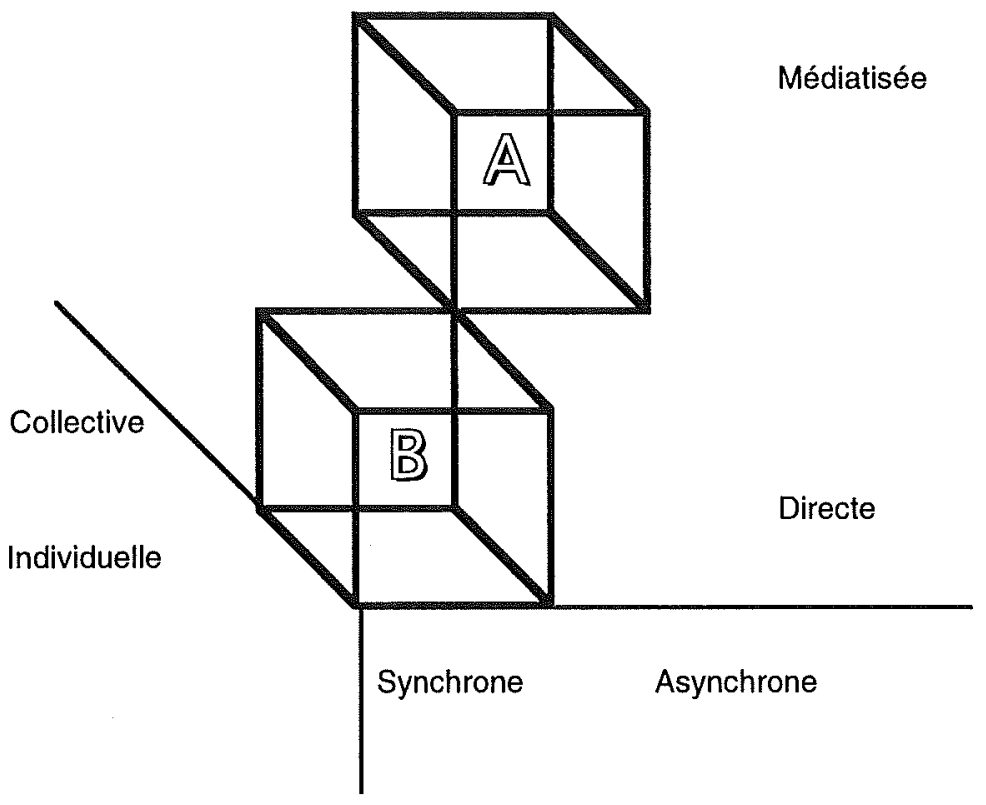

A: Conférence électronique (C.A.M.)

B: Encadrement particulier face à face (I.S.D.)

Schéma 2: Deux formes contrastées de communication pédagogique

b. la rapidité de la réponse: la qualité de l'interaction est directement dépendante de la rapidité de la réponse ${ }^{1}$;

c. la pertinence de la réponse : son adéquation à la question ou au problème soulevé par l'apprenant ; on se situe donc au plan de la matière et du contenu (modèle des connaissances);

d. l'individualisation de la réponse: son adéquation aux caractéristiques propres de l'apprenant ; on se situe donc au plan des connais-

1 Les études américaines montrent que, pour une banque de données informatique, un temps d'accès de 0,5 seconde est jugé satisfaisant par les utilisateurs (cf. AKSCYN et al., New York, 1988). 
connaissances personnelles de l'apprenant, de ses difficultés, de son degré de motivation, etc. (modèle de l'apprenant);

e. la flexibilité de l'échange : la capacité de l'apprenant à modifier le déroulement du processus d'assistance et à l'infléchir à tous moments selon ses besoins propres.

La présentation des critères généraux ainsi que de leurs variables permet de proposer une première analyse des différentes technologies de la communication -classiques et anciennes- potentiellement utilisables dans le cadre d'un système de FAD. A titre d'exemple, et pour mieux fixer les idées, le tableau ci-dessous présente quelques cas de figures fréquents, connus de tous:

\begin{tabular}{|l|l|l|l|l|}
\hline & \multicolumn{2}{|c|}{ RELATION TUTEUR:APPRENANT(S) } & $\begin{array}{l}\text { DEGRÉ } \\
\text { D'INTERACTIVITÉ }\end{array}$ \\
\hline COURRIER & Individuelle & Asynchronique & Médiatisée & Faible \\
\hline SUPERVISION & Individuelle & Synchronique & Directe & Forte \\
\hline $\begin{array}{l}\text { SÉANCE DE RE- } \\
\text { GROUPEMENT }\end{array}$ & Collective & Synchronique & Directe & Moyenne \\
\hline TÉLÉPHONE & Individuelle & Synchronique & Médiatisée & Forte \\
\hline FAX & Individuelle & Asynchronique & Médiatisée & Faible \\
\hline $\begin{array}{l}\text { CONFÉRENCE } \\
\text { TÉLÉPHONIQUE }\end{array}$ & Collective & Synchronique & Médiatisée & Fort \\
\hline E-MAIL & Individuel & Asynchronique & Médiatisée & Faible \\
\hline $\begin{array}{l}\text { ĆONFÉRENCE } \\
\text { ELETRONIQUE } \\
\text { CONFÉRENCE }\end{array}$ & Collective & Asynchronique & Médiatisée & Moyen \\
\hline
\end{tabular}

On le voit, ces différentes taxonomies se présentent toujours comme des instruments plus descriptifs qu'explicatifs, comme des listes de catégories empiriques dont les critères dépendent des centres d'intérêt des chercheurs et du regard qu'ils portent aux médias: apprentissage, fonctions d'enseignement, type de langage, modalité de communication, etc. Ces critères relèvent en définitive davantage du sens commun et du sens pratique que de règles scientifiques. Mais, en 
l'état actuel des connaissances, sans doute est-il difficile de faire autrement.

Dès lors, il paraît intéressant de proposer certaines orientations de recherches sans doute plus prometteuses et dont les résultats devraient intéresser les praticiens et de la formation présentielle et de la formation à distance : celles de la gestion discursive et de la planification de la communication pédagogique.

Une première piste est celle de l'étude, dans les document pédagogiques scriptovisuels conventionnels ou informatises des modalités d'insertion et d'exploitation de ce qu'il est convenu aujourd'hui d'appeller les paratextes: photographies, dessins, diagrammes, tableaux, notes en bas de page, formules mathématiques, etc. L'image et le texte ont fait chacun l'objet de nombreuses recherches psychopédagogiques: rôle de l'image dans l'apprentissage, analyse sémiotique, lisibilité, compréhension et mémorisation du texte, techniques de rédaction et d'écriture, etc. Pourtant, le rapport entre texte et paratexte n'a jamais été étudié systématiquement ${ }^{1}$.

$\mathrm{La}$ seconde problématique concernerait les relations entre les différentes formes de communication médiatisées $\left(\mathrm{M}^{+}\right)$ou non $\left(\mathrm{M}^{-}\right)$: le premier cas correspond à la situation de médiatisation technologique que nous avons suffisamment décrite tandis que le second correspond à la situation classique de communication orale -communication directe, face à face- émanant d'une personne, d'un locuteur quel que soit son rôle (formateur, éducateur, animateur, etc.). Or, dans la pratique, il est rare de trouver des situations de communication éducative qui ne relèveraient que d'une forme "pure". Les deux modes de production sont le plus souvent conjoints, selon les plusieurs modalités possibles:

1. la dépendance, réalisée sous forme d'un enchassement:

a. une forme médiatisée étant enchassée dans une forme forme nonmédiatisée $\left[\left(\mathrm{M}^{-}\right)^{[\mathrm{M}+]}\right]$ : par exemple, l'utilisation d'une illustration au sein d'un exposé oral;

1 Une recherche est actuellement en cours sur ce thème Modélisation des rappports texte paratexte dans les documents scripto-visuels (D. Peraya et M. Cl. Nyssen). Elle fera l'objet d'une publication dans les Cahiers de la Section des Sciences de l'Education de la Faculté de psychologie et des sciences de l'éducation de l'Université de Genève en décembre 1993. 
b. ou une forme non-médiatisée étant enchassée dans une forme médiatisée $\left[\left(\mathrm{M}^{+}\right)^{[\mathrm{M}-]}\right]$ : par exemple, un encadrement verbalisé d'un élève travaillant en auto-formation sur un logiciel d'EAO;

2. la complémentarité sous forme d'un fonctionnement alterné : l'aller retour permanent entre le commentaire verbal du formateur et l'image, la carte ou le tableau commenté $\left[\left(\mathrm{M}^{-}\right) \mathrm{vs}\left(\mathrm{M}^{+}\right) \mathrm{vs}\left(\mathrm{M}^{-}\right) \mathrm{vs}\right.$ $\left.\left(\mathrm{M}^{+}\right) \mathrm{vs}\left(\mathrm{M}^{-},\right)\right]$etc.

L'observation de la pratique pédagogique montre en effet que les ensiegnants ont souvent de la peine à réaliser ces formes d'intégration. Or, il est évident que dans la perspective globale d'une analyse de la gestion d'un cours, d'une séance d'animation ou d'information, les rapports de type $\left(\mathrm{M}^{-}\right) /\left(\mathrm{M}^{+}\right)$, considérés comme des rapports d'intertextualité ou de gestion intertextuelle, devraient retenir toute l'attention du chercheur. puisqu'ils concernent les formes de planification discursive. 